\title{
Das Engagement der Ärzteschaft ist gefragt
}

\section{Gisela Perren-Klingler}

Dr. med., Fachärztin für Psychiatrie und Psychotherapie, Mitglied FMH, Past President IHFFC

\author{
Die Autorin war während fünf Jahren Präsidentin der International Humanitarian \\ Fact Finding Commission (IHFFC). Anlass des folgenden Beitrags ist die zuneh- \\ mende Missachtung des Rechts auf Schutz für Verwundete, medizinisches Perso- \\ nal und die Zivilbevölkerung in Gebieten mit bewaffneten Konflikten. Die Ärzte- \\ schaft ist aufgerufen, sich in diesem Bereich zu engagieren.
}

Als im Oktober 2015 in Kunduz, Afghanistan, das Spital von Médecins sans Frontières (MSF) aus der Luft quasi total zerstört wurde, ist die IHFFC aktiv geworden: Wir haben den betroffenen Regierungen einen Brief geschrieben, in welchem wir unsere guten Dienste für eine unabhängige Untersuchung anboten. Während

\section{Es sind dabei Menschen getötet worden, die am verletzlichsten sind: Patienten in der Intensivstation und im Operationssaal.}

Afghanistan uns keine Antwort zukommen liess, hat das amerikanische Aussenministerium uns geantwortet, dass eine interne Untersuchung im Gange sei. In Kunduz ist ein grosses Spital von MSF aus der Luft angegriffen worden. Es sind dabei Menschen getötet worden, die am allermeisten verletzlich sind: Patienten in der Intensivstation und im Operationssaal und medizinisches Personal aus diesen beiden Abteilungen. Eine der Aufgaben des humanitären Völkerrechts (der Genfer Konventionen) ist, verwundete Kämpfer egal welcher Herkunft -, die Zivilbevölkerung und das Personal aus den medizinischen Berufen zu schützen. Das IKRK, das ja für die Verbreitung der Kenntnis darüber zuständig ist, bringt überall auf der Welt Armeen die Respektierung dieses Rechts theoretisch bei. Natürlich hängt dies auch von den Kommandanten und Befehlsgebern ab, doch der erste Schritt, die Kenntnis des Rechts, ist in allen regulären Armeen (und vielen Armee-ähnlichen Organisationen, wie z.B. der FARC und dem ELN in Kolumbien) bekannt. Das «Emblem», das Rote Kreuz oder der Rote Halbmond, ist klar geschützt.

Nach der Affäre Kunduz wurde mir immer klarer, dass genau dieses Recht, das die Leiden der Zivilbevölke- rung im Rahmen halten soll, an vielen Orten auf der Welt vorsätzlich missachtet wird: Im Jemen bombardiert die Allianz Spitäler, in Syrien sind über 200 Spitäler und andere medizinische Einrichtungen (z.B. Ambulatorien) zerstört worden, so dass man von einer medizinischen Notlage sprechen muss.

\section{Zurückhaltende Reaktionen seitens der Ärzteschaft}

Das IKRK, das an vorderster Front engagiert ist, hat deshalb die Arbeitsgruppe «Health Care in Danger» ins Leben gerufen; diese juristische und organisationelle

\section{Wer ist die IHFFC?}

Die International Humanitarian Fact Finding Commission (IHFFC) ist eine von den Mitgliedstaaten im Rahmen des I. Zusatzprotokolls der Genfer Konventionen geschaffene Kommission. Sie hat den Auftrag, bei bewaffneten Konflikten und bei Vorwürfen über Verletzungen des Kriegsrechtes (Genfer Konventionen) Fakten zu ergründen und dadurch dem Respekt der Genfer Konventionen Beachtung zu verschaffen.

Die Kommission wurde 1991 ins Leben gerufen; sie kann von den Staaten, die auch das Zusatzprotokoll angenommen und ratifiziert haben, relativ einfach anerkannt werden: Staaten, die die Kommission anerkannt haben, können für die alle fünf Jahre stattfindenden Wahlen einen Kandidaten zur Wahl stellen. Bis heute haben 76 Staaten die Kommission anerkannt.

Die Kommission hat verschiedene Besonderheiten: Sie ist gebunden durch die Verpflichtung, unabhängig, unparteiisch und neutral zu handeln; ihre Berichte müssen vertraulich an die betroffenen Parteien abgegeben werden. Sie ist weltweit die einzige Untersuchungskommission, die von den Regierungen getragen wird, d.h., sie ist keine NGO - oder UNO-Kommission. Die Kommission zählt genau 15 Mitglieder; die Schweiz stellt als Depositarstaat der Genfer Konventionen Personal aus dem EDA für das Sekretariat der Kommission zur Verfügung. Seit Beginn gab es immer ein Kommissionsmitglied aus der Schweiz. 


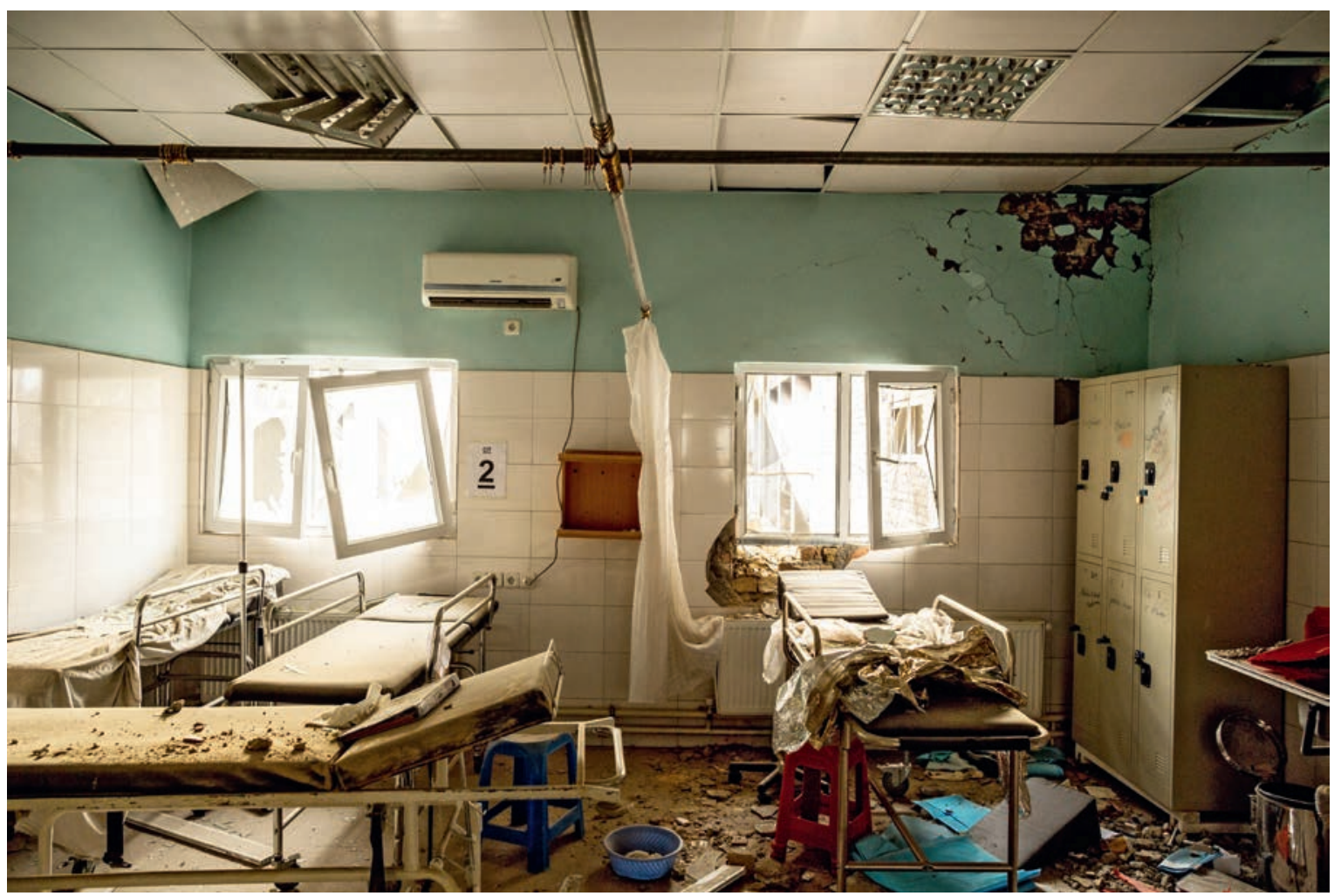

Am 3. Oktober 2015 wurde das Traumatologie-Spital von MSF im afghanischen Kundus bei einem Luftangriff des amerikanischen Militärs zerstört.

Arbeit wird wichtig sein. Im Nachgang zu Kunduz habe ich mich indes gewundert, wie wenig Reaktionen aus Gruppen von Ärzten in der ganzen Welt gekommen sind. Auch in persönlichen Gesprächen mit Kollegen, z.B. in Ashkalon (10 km von Gaza), war ich überrascht, wie selbstverständlich es für diese Israelis ist, dass ihr Spital unter Raketenbeschuss sein konnte (unterdessen tut ja der «Iron Dome» das Seine), und wie sie damit leben, dass - ausser dem Hort für die Kinder der Angestellten - nichts im Spital (nicht einmal die Operationssäle) geschützt ist. Man scheint einfach so zur Kenntnis zu nehmen und sich damit abzufinden, dass die Genfer Konventionen nicht respektiert werden.

\section{Respektierung von Gesetzen}

Respektierung eines Gesetzes wird nur durch das Wissen erreicht, dass nach dessen Verletzung eine Untersuchung stattfindet, die zu einer Verurteilung und zu einer Strafe führen kann.

Dazu braucht es jedoch auch einen Konsens in der betroffenen Bevölkerung. Die von den meisten Staaten anerkannten und ratifizierten Genfer Konventionen können nur (wieder?) besser respektiert werden, wenn gerade auch bei uns klargemacht wird, dass wir, Vertreter der Gesundheitsberufe, um unsere Arbeit profes- sionell auszuführen, in unseren Berufen auf diese Respektierung angewiesen sind. Auch unsere Schweizer Notfallteams kennen leider unterdessen die Verrohung der Sitten, wenn sie bei Notfalleinsätzen angegriffen werden. Auch darüber wird kaum gesprochen. Solange die Zivilgesellschaft, speziell aber unsere Berufsorganisationen, dies als unwesentlich ansieht, so-

\section{Man nimmt einfach so zur Kenntnis, dass die} Genfer Konventionen nicht respektiert werden.

lange dies nicht lautstark denunziert und in der öffentlichen Meinung bewusst gemacht wird, gibt es keine Hoffnung, der präventiven Funktion der Genfer Konventionen (im Sinn von Primär- und Sekundärprävention) Nachhaltigkeit zu verschaffen.

\section{Suche nach unterstützenden Massnahmen seitens der Ärzteschaft}

Dazu ist dieser Artikel für die SÄZ entstanden: Was können Schweizer Ärzte tun, um mit einer Bewusstmachung in unseren Berufsgruppen zu beginnen (Pflegepersonal im Allgemeinen). Das Ziel wäre, dass wir dann unsere Kollegen in Europa sensibilisieren, mit dem Ziel, die World Medical Association zu aktivieren. Wenn wir aus den helfenden Berufen es hinnehmen, 
Zehn Jahre in der International Humanitarian Fact Finding Commission (IHFFC)

2007 wurde ich in die Kommission gewählt und 2012 nach meinerWiederwahl intern zur Präsidentin der Kommission. Ich war die erste Nichtjuristin und die erste Frau im Präsidium. Als Präsidentin arbeitete ich mit einem Büro, das aus vier Vizepräsidenten besteht. In der Sicht der Kommission sollten die vier Vizepräsidenten aus verschiedenen Regionen der Welt kommen und verschiedene Berufsgruppen repräsentieren. In meinem ersten Büro standen mir zur Seite: ein Engländer, Jurist des britischen Militärs und Professor für humanitäres Völkerrecht in Cambridge; ein Emirati, Jurist, Richter und Professor an der Uni in Dubai; ein Uruguayer, Jurist der Flugwaffe; ein Japaner, Professor für Völkerrecht aus Tokyo.

\section{Überalterung gebremst}

Da ich selber keine Einführung von meinem Vorgänger erhalten hatte, setzte ich mich dafür ein, dass ein «President-elect» schon zwei Jahre vor den Neuwahlen designiert würde, um so eine gewisse Kontinuität im Büro zu garantieren und den designierten Präsidenten in die Arbeit einzuführen. Mein Vorschlag wurde nach langen Diskussionen angenommen; und so wurde 2015 der deutsche Jurist Thilo Marauhn zum «First Vice President» gewählt. Es war für mich auch von Anfang an klar gewesen, dass ich 2017 aus der Kommission ausscheiden würde, da ich während meiner Amtszeit immer wieder darauf hingewiesen hatte, dass wir überaltert seien und man eine Alterslimite respektieren sollte. Meine penetranten Bemerkungen hatten Erfolg, so dass für die Kommissionswahlen im Dezember 2016 die neun ältesten Mitglieder (inklusive mir) zurücktraten; es konnte also der Kommission ein jüngeres Gesicht mit Repräsentanten aus verschiedenen Berufen gegeben werden.

Schwarzafrika und Russland nicht vertreten

Leider konnte ich es nicht erreichen, der Kommission durch die Wahl eines Vertreters aus Schwarzafrika eine noch globalere Repräsentation zu geben. Obwohl drei qualifizierte Kandidaten aus Westafrika zur Wahl standen, wurde keiner gewählt, ebenso wenig wie der russische Kandidat.

Heute verfügt die Kommission über neun europäische Mitglieder, zwei Lateinamerikaner (Chile und Argentinien), zwei aus der Golfregion, einen Japaner und einen Algerier, d.h., mehr als die Hälfte der Mitglieder sind europäisch geprägt. Immerhin gibt es nun neben Juristen verschiedenster Spezialisierung drei Mediziner, einen Epidemiologen und zwei Diplomaten. Diese Diversifizierung wird die vorher ausschliesslich juristischen, häufig spitzfindigen Diskussionen zu mehr Praxisorientierung führen. Durch Fact Finding den Schutz von vulnerablen Gruppen zu verbessern, indem die Genfer Konventionen (das "Kriegsrecht») besser respektiert werden, ist ja etwas eminent Praktisches.

Ich werde nicht über meine vielen Aktivitäten, die ich während der fünf Jahre für die Kommission gehabt habe, berichten; bei Interesse kann dies auf der Website der Kommission (www.ihffc.org) abgefragt werden.

dass unser Emblem, das Rote Kreuz oder der Rote Halbmond, missachtet wird und Spitäler «zufälligerweise», als Kollateralschaden «für Wichtigeres» getroffen werden, wird sich nichts ändern und die Genfer Konvention eigentlich de facto annulliert. Es gilt, langfristig neu ein Tabu aufzurichten, das sagt, dass das Emblem geschützt werden muss; und dass, wenn dies nicht der Fall ist, eine Untersuchung sofort in Gang gesetzt wird, die unabhängig, unparteiisch und neutral zuerst die Tatsachen eruiert. Dabei könnte die IHFFC eine Hilfe bedeuten. Sie würde dann einen vertraulichen Bericht an die betreffenden Staaten erstellen. Dann liegt es an diesen, ihre (Militär-)Justiz in Gang zu setzen, um eine Strafuntersuchung (mit eventuellem Urteil im Anschluss) in die Wege zu leiten. Damit würde die nationale Rechtsprechung gestärkt und die (vielerorts als kolonialistisch und nicht unabhängig wahrgenommene) Macht der UNO-Kommissionen in einen anderen Rahmen gesetzt.

Selbstverständlich würde dies leider nicht bedeuten, dass der Internationale Strafgerichtshof in Den Haag keine Arbeit mehr hätte, da es ja sicher weiterhin Regierungen geben wird, welche Missachtung der Genfer Konventionen national nicht verfolgen. Doch es wäre möglich, in der Weltzivilbevölkerung eine öffentliche Meinung herzustellen, die besagt, dass es Rechte gibt, für die eingestanden werden muss. Die Macht der Ärzteorganisationen als Meinungsmacher könnte hier wieder einmal dargestellt werden, ausserhalb der Tarif- und Bezahlungskämpfe. Und auf eine längere Sicht wird es hoffentlich das Schicksal der hoch vulnerablen Gruppen, der Zivilbevölkerung (inklusive Kinder!), der Kriegsgefangenen, der Kranken und Verletzten, sicherer machen, ebenso wie der helfenden Berufe.

\section{Aufruf und Bitte}

Mein Aufruf geht also an alle Kolleginnen und Kollegen, die bereit sind, sich zu organisieren, um ein Komitee zum Schutz der Genfer Konventionen zu gründen. Die Aufgabe des Komitees wäre es dann, unsere europäischen Kollegen zu aktivieren und über deren Berufsorganisationen schliesslich in einer Art Schneeballphänomen bis zur Aktivierung der World Medical Association zu kommen; sicher wird es an einem gewissen Punkt auch zu einer Koordination mit den Aktivitäten des IKRK zu Health Care in Danger und mit MSF kommen müssen; dabei sollte wieder die Schweizerische Ärztegesellschaft federführend sein, gibt es doch wohl nirgends sonst so viele Ärzte, die einmal für das IKRK aktiv waren, und ist doch der Sitz des IKRK in Genf nahe bei Bern.

Mich mit diesem Thema zu befassen war mir während meiner Präsidentschaft nicht möglich - einerseits wegen der Verpflichtung zu Neutralität, andererseits wegen der bereits beträchtlichen Arbeitslast durch die Kommission. Nun aber ist dies eine Obsession, für die ich bereit bin, nochmals Energie einzusetzen.

Übrigens: Die Kommission hat am 19.5.2017 den ersten Auftrag erhalten, eines meiner ausgesprochenen und anvisierten Ziele während meiner Präsidentschaft. In der Ostukraine sollen die Tatsachen in Bezug auf den Tod eines medizinischen Helfers und die Verletzungen anderer aus der OSZE - Mission untersucht werden. Das ist ein Hoffnungsschimmer, der allerdings die Aktivierung der Ärzteschaft nicht unnötig macht!

Bildnachweis

(C) Andrew Quilty 